\title{
Ascorbic Acid and Chronic Alcohol Consumption in the Guinea Pig ${ }^{1}$
}

\author{
Robert L. Susick, Jr., ${ }^{*}$ Gerald D. Abrams, $†$ Christine A. Zurawski, \\ AND VINCENT G. ZANNONI* \\ Departments of * Pharmacology and $\dagger$ Pathology, The University of Michigan \\ Medical School, Ann Arbor, Michigan 48109
}

Received November 15, 1985: accepted January 29, 1986

\begin{abstract}
Ascorbic Acid and Chronic Alcohol Consumption in the Guinea Pig. SusICK, R. L., JR., ABRAMS, G. D., ZuRawsKI, C. A., AND ZanNoni, V. G. (1986). Toxicol. Appl. Pharmacol. 84, 329-335. Protection against the toxic effects of chronic alcohol consumption was observed in male guinea pigs maintained on a high-ascorbic-acid diet (vitamin $\mathrm{C}$-deficient chow plus $2.0 \mathrm{mg}$ ascorbic $\mathrm{acid} / \mathrm{ml}$ drinking water) as compared to animals on a low-ascorbic-acid diet (vitamin C-deficient chow and from 0.025 to $0.050 \mathrm{mg}$ ascorbic acid/ml drinking water). Alcohol was orally administered to the guinea pigs at a dose of $2.5 \mathrm{~g} / \mathrm{kg}$ for up to 14 weeks. Levels of serum aspartate aminotransferase and serum alanine aminotransferase were significantly elevated in animals on the low-ascorbicacid diet that received alcohol, 120 and $250 \%$, respectively. In contrast, in animals on the highascorbic-acid diet that received alcohol, levels of alanine aminotransferase were not significantly elevated and levels of aspartate aminotransferase were elevated $50 \%$. In addition, some of the animals on the low-ascorbic-acid diet that received alcohol for 12 to 14 weeks developed hepatic steatosis and necrosis, whereas none of the animals on the high-ascorbic-acid diet that received alcohol for the same length of time manifested these changes. (c) 1986 Academic Press, Inc.
\end{abstract}

Although alcohol adversely affects almost every biological system in the body, the primary target organ for alcohol toxicity remains the liver. It is well established that the earliest and most common response to chronic alcohol ingestion is the development of hepatic steatosis (Goldstein, 1983; Lieber, 1982). Increased levels of serum enzymes such as aspartate aminotransferase (SGOT) ${ }^{2}$ and alanine aminotransferase (SGPT), ${ }^{3}$ which are indices of increased permeability and/or necrosis of hepatocytes, have also been observed after alcohol consumption (Goldberg and Watts, 1965; Lieber, 1982). Chronic exposure to large amounts of alcohol can eventually lead to cir-

\footnotetext{
${ }^{1}$ This work was supported in part by Grant 23007 from Hoffmann-La Roche, Inc., Nutley, N.J.

${ }^{2}$ Aspartate aminotransferase $=$ serum glutamic oxaloacetic transaminase (SGOT).

${ }^{3}$ Alanine aminotransferase $=$ serum glutamic pyruvic transaminase (SGPT).
}

rhosis and death. In addition, alcohol has been demonstrated to induce specific forms of cytochrome P-450 (Ohnishi and Lieber, 1977; Ishii et al., 1973).

The ability of ascorbic acid to protect against the toxicity of ethanol has been studied to a limited extent. Yunice and Lindeman (1977) found that pretreatment with ascorbic acid protected against the lethality of an acute dose of ethanol in mice and against the lethality of chronic ethanol exposure in the rat. Yunice et al. (1984) also found less hepatic steatosis in animals on high-ascorbic-acid diet than in animals on a low-ascorbic-acid diet after 8 weeks of ethanol infusion. In addition, ascorbic acid has been shown to reverse the impaired swimming behavior of mice treated with an intoxicating dose of ethanol (Busnel and Lehmann, 1980) and to prevent the marked depletion of circulating eosinophils in the rat caused by alcohol (Vanha-Perttula, 1960). 
This study examines the ability of dietary ascorbic acid to protect against liver damage caused by prolonged alcohol consumption. Guinea pigs on diets either low or high in the vitamin were force-fed $2.5 \mathrm{~g}$ ethanol $/ \mathrm{kg}$ body weight 5 days per week for up to 14 weeks. Histopathologic study of the liver was performed as well as the determination of SGOT and SGPT levels. The quantity of cytochrome $P-450$ and flavin-monooxygenase activity, two important systems involved in the catalysis of xenobiotics, were also assessed after chronic alcohol consumption. Levels of ascorbic acid in the liver were determined.

\section{METHODS}

Source of chemicals, materials, and guinea pigs. LAscorbic acid and the materials for the determination of SGOT and SGPT levels were purchased from Sigma Chemical Company (St. Louis, Mo.). Dimethylaniline and $\alpha, \alpha$-dipyridyl were purchased from Fischer Scientific Company (Fairlawn, N.J.) Ninety-five percent ethanol was used to make all alcohol solutions. Other chemicals used were of reagent grade or better. Ascorbic-acid-deficient guinea pig chow was purchased from Nutritional Biochemicals (Cleveland, Ohio). Male Hartley guinea pigs (225 to $275 \mathrm{~g}$ ) were purchased from the Michigan Department of Public Health (Lansing, Mich.).

Animal treatment. Male Hartley guinea pigs (225 to 275 g) were housed at $22 \pm 2{ }^{\circ} \mathrm{C}$ on a 12 -hr light cycle. The animals were divided into two groups; one group received a diet low in ascorbic acio ( 9 animals) and the other group a diet high in ascorbic acid ( 13 animals). The low-ascorbicacid diet consisted of vitamin-C-deficient guinea pig chow and from 0.025 to $0.050 \mathrm{mg}$ ascorbic acid/ml drinking water. The drinking water containing ascorbic acid was changed each day. The ascorbic acid is stable for at least 2 days as determined by the method of Zannoni $e t$ al. (1974). The exact concentration of ascorbic acid in the drinking water was modulated to prevent the animals from becoming frankly scorbutic and to maintain a growth rate similar to those of animals on the high-ascorbic-acid diet. The high-ascorbic-acid diet consisted of vitamin-C-deficient guinea pig chow and $2.0 \mathrm{mg}$ ascorbic acid/ml drinking water. The animals were maintained on these diets for 3 weeks prior to ethanol administration. The normal daily intakc of ascorbic acid for the guinea pig is 2 to $3 \mathrm{mg} /$ day. This amount of the vitamin will result in hepatic concentrations of 70 to $80 \mathrm{mg} / 100 \mathrm{~g}$ hepatic protein (Zannoni et al., 1974). The low-ascorbic-acid regimen was chosen to provide the animals with enough of the vitamin to prevent them from becoming frankly scorbutic (no weight loss and no or minimal hemorrhages) and with hepatic concentrations of the vitamin from 20 to $40 \mathrm{mg}$ / $100 \mathrm{~g}$ hepatic protein.

Ethanol was force-fed to the animals as a $50 \%$ solution in water 5 days per week up to, but not including, the day of termination. A syringe, capped with a short rubber tube, was used to feed the alcohol to the animals. The first dose given was $0.4 \mathrm{~g} / \mathrm{kg}$, to familiarize the animals with the taste of alcohol, and the dose was gradually increased to $2.5 \mathrm{~g} / \mathrm{kg}$ over a 2-week period. Animals were weighed three times per week and killed mid-week at intervals between 6 and 14 weeks. One animal from the low-ascorbic-acid group was killed at 6,7 , and 11 weeks and two animals at 10,12 , and 14 weeks. Two animals from the high-ascorbic-acid group were killed at $6,10,11,12$, and 14 weeks and three at 7 weeks. Histology, SGOT, SGPT, cytochrome $P-450$, flavin-monooxygenase activity, and hepatic ascorbic acid concentrations were determined for each animal in the study. For all of the parameters mentioned above, excluding histology, length of alcohol exposure had no significant effect on the values obtained within each group. For this reason, the animals in the low-ascorbic-acid group which received alcohol were treated as one group, and the animals in the high-ascorbic-acid group which received alcohol were treated as one group with respect to the biochemical parameters measured. Mean values for SGOT, SGPT, $P-450$, flavin-monooxygenase activity, and hepatic ascorbic acid concentrations for the two ascorbic acid groups were thus obtained and compared.

Animals were decapitated, liver slices were placed in $10 \%$ buffered neutral Formalin for pathological examination, and the remaining liver was homogenized in 4 volumes of cold $0.1 \mathrm{M}$ sodium phosphate buffer, $\mathrm{pH} 7.4$. The hepatic tissue homogenates were frozed at $-12^{\circ} \mathrm{C}$ for protein and ascorbic acid determination. Twenty milliliters of the $20 \%$ liver homogenate were centrifuged at $12,000 \mathrm{~g}$ for $30 \mathrm{~min}$. The $12,000 \mathrm{~g}$ supernatant fraction was removed and frozen for the determination of the quantity of cytochrome $P-450$ and flavin-monooxygenase activity.

Two groups of control animals were utilized, six on the low-ascorbic-acid regimen and six on the high-ascorbicacid regimen. These animals were treated identically to the experimental animals but did not receive alcohol. They were killed after 14 weeks of receiving the ascorbic-aciddeficient diet plus ascorbic acid supplements.

Histological studies of the liver. Liver slices which had been placed in $10 \%$ buffered neutral Formalin were paraffin-embedded, sectioned at $5 \mu \mathrm{m}$, and then stained with hematoxylin and eosin. The sections were examined by the pathologist (G.D.A.) The degree of fatty infiltration was estimated by assessment of the vacuolar change evident with hematoxylin and eosin staining.

Biochemical and enzymological assays. The serum enzymes, aspartate aminotransferase and alanine aminotransferase, were determined according to the established method of Reitman and Frankel (1957). The quantity of cytochrome $P-450$ was determined by the method of 
Omura and Sato (1964). Flavin-monooxygenase activity was determined by measuring $N$-oxidation of dimethylaniline according to the method of Ziegler and Pettit (1964). Hepatic ascorbic acid concentrations were determined by the method of Zannoni et al. (1974). Protein was determined by the method of Lowry et al. (1951) with bovine serum albumin as the standard.

Statistical analysis. Statistical significance of the data was determined with the two-sided Student's $t$ test by comparing means. Differences were considered significant at a $p$ value of less than 0.05 .

\section{RESULTS}

Animals on the low- and high-ascorbic-acid diets that received alcohol gained weight at the same rate over the course of the study (Fig. 1). Control animals on the low- and highascorbic-acid diets gained weight at the same rate as the alcohol-treated animals for the first 5 weeks and at a faster rate $(35 \%)$ during the remaining weeks of the study.

Serum enzymes. Levels of SGOT and SGPT, which were measured at the time of termination, were significantly elevated in the animals on the low-ascorbic-acid diet that received alcohol (Table 1). The mean SGOT value for animals on the low-ascorbic-acid diet that received alcohol was increased $120 \%$ over control values and the mean SGPT value was

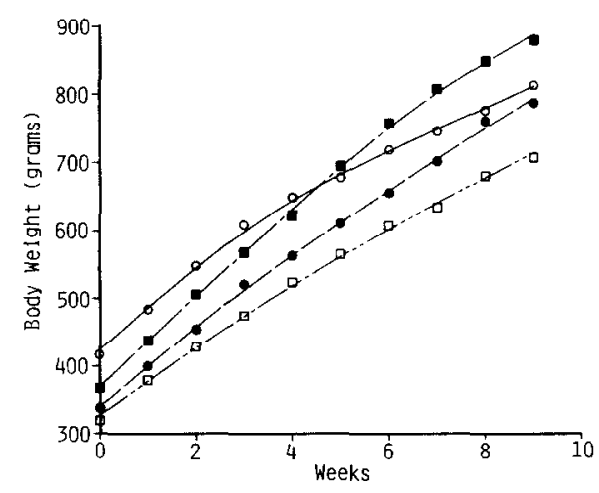

FIG. 1. Growth curves of animals that received alcohol and their controls: ( ) low-ascorbic-acid diet without alcohol; (O) low-ascorbic-acid diet with alcohol; ( $($ ) highascorbic-acid diet without alcohol; $(\square)$ high-ascorbic-acid diet with alcohol. Each point represents the mean of six animals. All other animals within each group had similar growth rates. increased $250 \%$. In marked contrast, animals on the high-ascorbic-acid diet that received alcohol showed no significant increase in SGPT levels and only a $50 \%$ increase in SGOT levels. Chronic alcohol consumption had no effect on liver weight to body weight ratios in either the low- or high-ascorbic-acid groups.

Hepatic ascorbic acid concentrations. Animals on the low-ascorbic-acid diet that received alcohol had a $32 \%$ lower mean hepatic ascorbic acid concentration than animals on the low-ascorbic-acid diet that did not receive alcohol, $20 \pm 6(8) \mathrm{mg} / 100 \mathrm{~g}$ protein versus $30 \pm 9(6) \mathrm{mg} / 100 \mathrm{~g}$ protein, $p<0.05$. There was no significant difference in the hepatic concentration of the vitamin in animals on the high-ascorbic-acid diet which received alcohol when compared to animals on the highascorbic-acid diet that did not receive alcohol, $120 \pm 28$ (13) versus $130 \pm 34(6) \mathrm{mg} / 100 \mathrm{~g}$ protein.

Cytochrome P-450 and flavin-monooxygenase activity. There was no significant difference in the quantity of cytochrome $P-450$ in the animals on the two ascorbic acid diet regimens that did not receive alcohol (Table 2). The alcohol-treated animals, however, on both the low- and high-ascorbic-acid diets had significantly higher quantities of cytochrome $P-450$ than their controls; 2.6 -fold and 1.8fold, respectively.

In contrast to the increased levels of cytochrome $P-450$ found in animals that received alcohol, chronic alcohol consumption had no effect on the flavin-monooxygenase activity in animals on the low-ascorbic-acid diet and a $47 \%$ inhibitory effect on flavin-monooxygenase activity in animals on the high-ascorbic-acid diet, $P<0.05$.

Histopathology. There were no significant histologic abnormalities in the livers from animals on either the low- or high-ascorbic-acid diets that did not receive alcohol. Of the four livers examined from the low-ascorbic-acid group receiving alcohol for 12 to 14 weeks, however, two had steatosis. One of these was rated as severe (Fig. 2A) and the other moderate. Steatosis was not seen in the four cor- 
TABLE 1

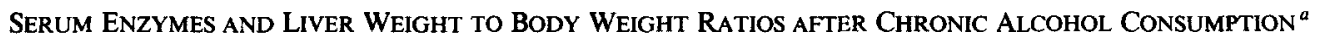

\begin{tabular}{|c|c|c|c|c|}
\hline & \multicolumn{2}{|c|}{ Low-ascorbic-acid diet ${ }^{b}$} & \multicolumn{2}{|c|}{ High-ascorbic-acid diet ${ }^{c}$} \\
\hline & No alcohol & Alcohol & No alcohol & Alcohol \\
\hline SGOT (units/ml) ${ }^{d}$ & $42 \pm 8(5)$ & $92 \pm 34(8)^{* *}$ & $32 \pm 12(6)$ & $47 \pm 12(12)^{*}$ \\
\hline SGPT (units/ml) ${ }^{d}$ & $15 \pm 9(5)$ & $52 \pm 29(8)^{*}$ & $14 \pm 5(6)$ & $24 \pm 11(12)$ \\
\hline Liver weight/body weight (mg/g) & $41 \pm 3(6)$ & $42 \pm 10(9)$ & $43 \pm 7(6)$ & $38 \pm 10(13)$ \\
\hline
\end{tabular}

${ }^{a}$ Values represent the $\bar{x} \pm$ SD with number of animals in parentheses.

${ }^{b}$ Mean hepatic ascorbic acid concentration: no alcohol, $30 \pm 9 \mathrm{mg} / 100 \mathrm{~g}$ protein; alcohol, $20 \pm 6$.

${ }^{c}$ Mean hepatic ascorbic acid concentration: no alcohol, $130 \pm 34 \mathrm{mg} / 100 \mathrm{~g}$ protein; alcohol, $120 \pm 28$.

${ }^{d} 1$ unit $=4.82 \times 10^{-4} \mu \mathrm{mol}$ glutamate formed $/ \mathrm{min}$.

$* p<0.05$ from nonalcohol control group.

** $p<0.01$ from nonalcohol control group.

responding high-ascorbic-acid animals receiving alcohol for the same period of time (Fig. 2B). Two of the four low-ascorbic-acid animals also had patchy necrosis of hepatocytes (not in a zonal distribution), while none of the highascorbic-acid animals manifested this change. No hepatic abnormalities were evident in any of the animals which received alcohol for less than 12 weeks.

\section{DISCUSSION}

Elevation in SGOT and SGPT levels is indicative of increased permeability and/or ne- crosis of hepatocytes. Ethanol is known to disorder membranes, expanding their area, making them more fluid, and increasing the mobility of membrane components (Chin and Goldstein, 1977). Ethanol is also a direct hepatotoxicant resulting in necrosis of hepatocytes (Goldstein, 1983; Lieber, 1982).

Fat accumulation in liver cells is caused by a number of factors, two of which result from the oxidation of alcohol itself via alcohol dehydrogenase. When alcohol is oxidized by alcohol dehydrogenase, an increase in the concentration of NADH occurs, and the cellular redox balance shifts to the reduced state. One

TABLE 2

CYTOCHROME $P-450$ QUANTITY AND FLAVIN-MONOOXYGENASE ACTIVITY AFTER CHRONIC ALCOHOL CONSUMPTION ${ }^{a}$

\begin{tabular}{|c|c|c|c|c|}
\hline & \multicolumn{2}{|c|}{ Low-ascorbic-acid diet ${ }^{b}$} & \multicolumn{2}{|c|}{ High-ascorbic-acid diet ${ }^{c}$} \\
\hline & No alcohol & Alcohol & No alcohol & Alcohol \\
\hline Cytochrome $P-450^{d}$ & $2.5+0.5(6)$ & $6.5+3.3(8)^{*}$ & $4.5+2.2(6)$ & $8.3+1.9(13)^{* *}$ \\
\hline Flavin-monooxygenase $e^{e}$ & $1.3+0.7(6)$ & $0.7+0.3(8)$ & $1.7+0.8(6)$ & $0.9+0.4(12)^{*}$ \\
\hline
\end{tabular}

${ }^{a}$ Values represent the $\bar{x}+\mathrm{SD}$ with number of animals in parentheses.

${ }^{b}$ Mean hepatic ascorbic acid concentration: no alcohol, $30+9 \mathrm{mg} / 100 \mathrm{~g}$ protein; alcohol, $20 \pm 6$.

${ }^{c}$ Mean hepatic ascorbic acid concentration: no alcohol, $130 \pm 34 \mathrm{mg} / 100 \mathrm{~g}$ protein; alcohol, $120 \pm 28$.

${ }^{d} \mathrm{nmol} / 100 \mathrm{mg}$ protein.

$\mathrm{nmol} / \mathrm{min} / \mathrm{mg}$ protein.

* $p<0.05$ from the nonalcohol control group.

** $p<0.01$ from the nonalcohol control group. 


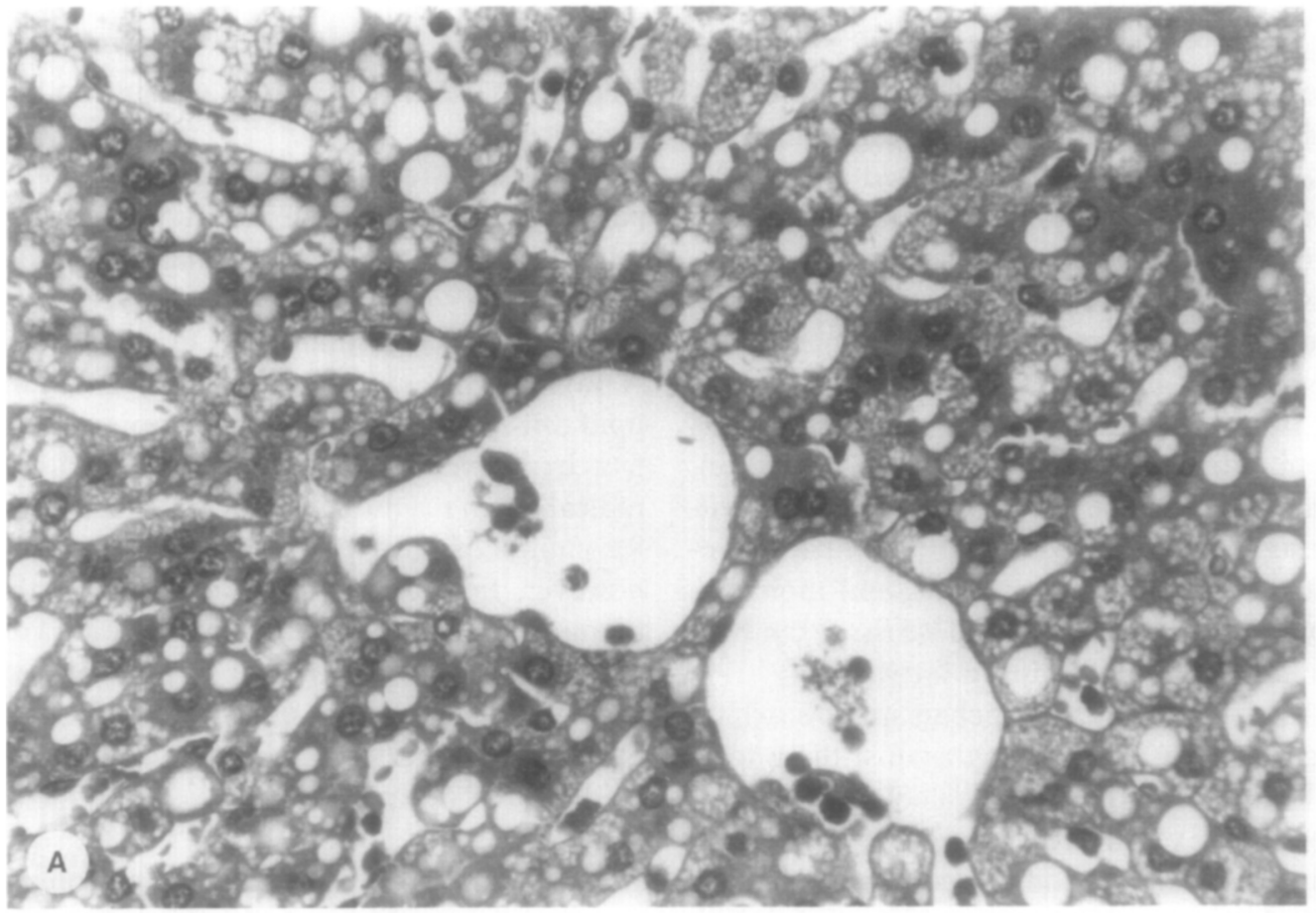

FIG. 2(A). Photomicrograph of the liver (hematoxylin and cosin, $\times 376$ ) from animal on the low-ascorbicacid diet, after 14 weeks of ethanol feeding. Note the marked, diffuse vacuolar change in hepatocytes, indicative of steatosis.

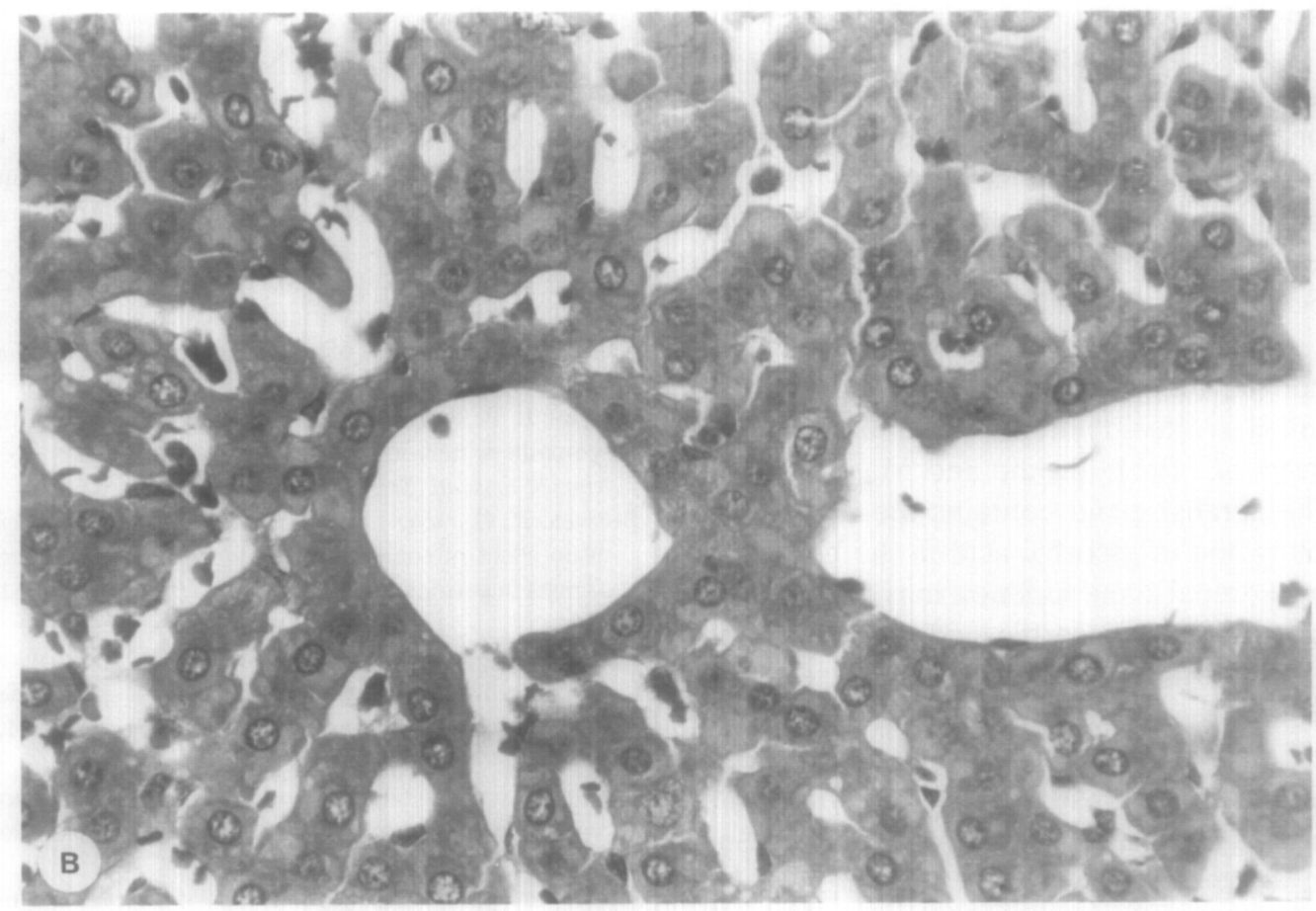

FIG. 2(B). Photomicrograph of the liver (hematoxylin and eosin, $\times 376$ ) from animal on the high-ascorbicacid diet, after 14 weeks of ethanol feeding. In contrast to Fig. $2 \mathrm{~A}$, there is no steatosis. 
avenue for the liver to dispose of the excess reducing equivalents is to synthesize more lipids. The excess NADH generated also results in decreased lipid oxidation. With progressive liver injury, lipoprotein secretion may fall below normal levels, thus aggravating fat accumulation and liver injury even further (Goldstein, 1983). Ethanol is also capable of causing a stress-induced mobilization of fatty acids from adipose tissue (Brodie and Maickel, 1963). All of these factors, increased lipid synthesis, decreased lipid oxidation, impaired hepatic secretion of lipoproteins, and mobilization of fatty acids from adipose tissue contribute to the ethanol-induced fatty liver.

The biochemical mechanism by which ascorbic acid is protecting against these toxicological consequences of alcohol consumption is as yet unclear. Several possibilities, however, can be considered. Protection by ascorbic acid against elevated levels of SGOT and SGPT indicate that the vitamin may be stabilizing the cellular membrane. In regard to this, the vitamin has been demonstrated to prevent permeability of epithelial cells of mucosal tissue (Alfano et al., 1975), and in preventing capillary fragility and permeability of the peripheral vascular system (Lee, 1961). Protection of the cellular membrane may also result in protection of the lipid secretory processes that are dependent on membrane integrity, thus diminishing fat accumulation.

Ascorbic acid may also minimize the stressinduced mobilization of fatty acids which is linked to altered adrenal gland function (Brodie et al., 1961; Brodie and Maickel, 1963). The adrenal gland contains the highest concentration of ascorbic acid in the body. Normal adrenal gland function may be dependent upon this high ascorbic acid concentration.

In addition, ascorbic acid may be able to prevent the increased $\mathrm{NADH}$ to $\mathrm{NAD}^{+}$ratio, responsible for increased lipid synthesis and decreased lipid oxidation, by providing an alternate pathway for alcohol oxidation, Such an alternate pathway has been proposed by Susick and Zannoni (1984), who have described an ascorbic-acid-dependent alcohol- oxidizing system that utilizes catalase to oxidize alcohol.

Lipid antioxidants have been shown in the past to prevent or reduce hepatic steatosis in rats after both acute and chronic alcohol administration (DiLuzio, 1964; Lieber and DeCarli, 1966; Hartman and DiLuzio, 1968). A proposed mechanism was advanced that the lipid antioxidants exerted their protective effect by preventing an ethanol-induced free radical chain reaction which would lead to the formation of lipid peroxides. It is unlikely, however, that ascorbic acid is acting in this manner. Although the vitamin is an antioxidant, it has not been shown to prevent lipid peroxidation. To the contrary, ascorbic acid has actually been found to enhance lipid peroxidation (Sato and Zannoni, 1976; Ohel et al., 1985).

It will be of interest to determine the precise biochemical mechanism of the protective action of ascorbic acid against alcohol toxicity and to determine if such protection can occur in man.

\section{REFERENCES}

Alfano, M. C., Miller, S. A., AND Drummond, J. F. (1975). In Second Conference on Vitamin C (C. G. King and J. J. Burns, eds.), Vol 258, p. 253. N.Y. Acad. Sci., New York.

Brodie, B. B., Butler, W. M., AND HORNING, M. G. (1961). Alcohol-induced triglyceride deposition in liver through derangement of fat transport. Amer. J. Clin. Nutr. 9, 432-435.

Brodie, B. B., AND MAICKEL, R. P. (1963). Role of the sympathetic nervous system is drug-induced fatty liver. Ann. N.Y. Acad. Sci. 104, 1049-1058.

BusNel, R. G., AND LehmanN, A. G., (1980). Antagonistic effect of sodium ascorbate on ethanol-induced changes in swimming of mice. Behav. Brain Res. 1, 351356.

ChIN, J. H., AND Goldstein, D. B. (1977). Effect of low concentrations of ethanol on the fluidity of spin-labeled erythrocyte and brain membranes. Mol. Pharmacol. 13, 435-441.

DiLuziU, N. R. (1964). Prevention of the acute ethanolinduced fatty liver by the simultaneous administration of antioxidants. Life Sci. 3, 113-118.

GoldBerG, D. M., AND WAT'S, L. (1965). Serum enzyme changes as evidence of liver reaction to oral alcohol. Gastroenterology 49, 256-261. 
Goldstein, D. B. (1983). Pharmacology of Alcohol. Oxford Univ. Press, New York.

HARTMAN, A. D., AND DiLuZIO, N. R. (1968). Inhibition of the chronic ethanol-induced fatty liver by antioxidant administration. Proc. Soc. Exp. Biol. Med. 127, 270 276.

ISHII, H., JOLY, J. G., AND LIEBER, C. S. (1973). Effect of ethanol on the amount and enzyme activities of hepatic rough and smooth microsomal membranes. Biochim. Biophys. Acta 291, 411-420.

LEE, R. E. (1961). In Vitamin C (J. J. Burns, ed.), Vol. 92, p. 295. N.Y. Acad. Sci., New York.

LIEBER, C. S. (1982). Medical Disorders of Alcoholism: Pathogenesis and Treatment. Saunders, Philadelphia.

LIEBER, C. S., AND DECARLI, L. M. (1966). Study of agents for the prevention of the fatty liver produced by prolonged alcohol intake. Gastroenterology 50, 316-322.

Lowry, O., Rosebrough, N. J., FARR, A. L., AND RaNDALL, R. J. (1951). Protein determination with the Folin phenol reagent. J. Biol. Chem. 193, 265-275.

OHEl, G., Kisselevitz, R., Margalioth, AND SCHENKER, J. G. (1985). Ascorbate-dependent lipid peroxidation in the human placenta and fetal membranes. Gynecol. Obstet. Invest. 19, 73-77.

OHNISHI, K., AND LIEBER, C. S. (1977). Reconstitution of the microsomal ethanol oxidizing system (MEOS): Qualitative and quantitative changes of cytochrome P450 after chronic ethanol consumption. J. Biol. Chem. 252, 7124-7131.

OMURA, T., AND SATO, R. (1964). The carbon monoxide- binding pigment of liver microsomes. I. Biol. Chem. 239, 2370-2378.

ReITMAN, S., AND FranKEL, S. (1957). A colorimetric method for the determination of serum glutamic oxalacetic and glutamic pyruvid transaminases. Amer. $J$. Clin. Pathol. 28, 56.

Sato, Ph. H., and Zannoni, V. G. (1976). Ascorbic acid and hepatic drug metabolism. J. Pharmacol. Exp. Ther. 198, 295-307.

SUSICK, R. L., JR., AND ZANNONI, V. G. (1984). Ascorbic acid and alcohol oxidation. Biochem. Pharmacol. 24, 3963-3969.

VANHa-Perttula, T. P. J. (1960). The influence of vitamin $\mathrm{C}$ on eosinophil response to acute alcohol intoxication in rats. Acta Endocrinol. 35, 585-593.

Yunice, A. A., Hsu, J. M., FAHMY, A., AND HeNRY, S. (1984). Ethanol-ascorbate interrelationship in acute and chronic alcoholism in the guinea pig. Proc. Soc. Exp. Biol. Med. 177, 262-271.

Yunice, A. A., AND LINDEMAN, R. D. (1977). Effect of ascorbic acid and zinc sulfate on ethanol toxicity and metabolism. Proc. Soc. Exp. Biol. Med. 154, 146-150.

ZANNONI, V. G., LYNCH, M., GoldSTEIN, S., AND SATO, P. (1974). A rapid micromethod for the determination of ascorbic acid in plasma and tissue. Biochem. Med. 11, $41-48$.

Ziegler, D. M., ANd Pettit, F. H. (1964). Formation of an intermediate $N$-oxide in the oxidative demethylation of $N, N$-dimethylaniline catalyzed by liver microsomes. Biochem. Biophys. Res. Commun. 15, 188-193. 